\title{
Consonâncias e Dissonâncias Interpretativas no Inter-relacionamento das Variáveis da Corrente Pulsada Aplicada à Soldagem com arame de Alumínio 4043
}

\author{
(Interpretative Agreements and Disagreements in the inter-relationships of the variables of the pulsed current applied to the \\ aluminum wire 4043)
}

\author{
Jair Carlos Dutra', Cleber Marques², Régis Henrique Gonçalves e Silva ${ }^{3}$ \\ ${ }^{1,2,3}$ Universidade Federal de Santa Catarina, Departamento de Engenharia Mecânica, Laboratório de Soldagem, Florianópolis, Santa \\ Catarina,Brasiljdutra@labsolda.ufsc.br ${ }^{1}$,cmarques@labsolda.ufsc.br ${ }^{2}$, regis@labsolda.ufsc.br ${ }^{3}$
}

\section{Resumo}

Na sua essência, o objetivo fundamental da utilização da corrente pulsada em soldagem é aumentar a relação potência do processo/ velocidade do arame, já que a mesma é muito baixa na transferência por curto circuito. Ocorrem, por isso, soldas com baixa qualidade, principalmente em alumínio e suas ligas e em aços inoxidáveis. Com a corrente pulsada, evitando-se os curtos-circuitos, a relação potência/velocidade do arame aumenta, pois a tensão nunca atinge valores abaixo de $15 \mathrm{~V}$. Então, a lógica de controle é tão somente evitar o curto circuito, sendo, portanto, ilógico a conjectura de sistemas com mais de uma gota por período. O que é vital para a técnica da pulsação é o arranjo físico das variáveis da onda. Por isso, este artigo analisa vários conjuntos de corrente de pulso e tempo de pulso, constituindo diferentes parâmetros de destacamento, e também diferentes diâmetros de gota. É analisado o relacionamento entre as referidas variáveis, considerando o expoente da corrente de pulso igual a 2,3, segundo o pesquisador Amin, e o expoente 2,0, segundo a maioria das adoções da literatura. Foi encontrado, entretanto, o valor de 1,83 como o melhor ajuste para o arame ER4043. Foi demonstrado também que a melhor condição de destacamento é quando esta ocorre logo depois da fase de pulso. Além disso, foi comprovado que o coeficiente de estabilidade $(\varphi)$ proposto por Gosh não atende aos objetivos propostos.

Palavras-chave: parâmetro de destacamento, destacamento de gotas, soldagem de alumínio.

\begin{abstract}
In essence, the fundamental purpose of the use of pulsed current welding is to increase the relationship power of the process / wire speed, since it is very low in the short circuit transfer. Therefore, welds result in poor quality, especially on aluminum and its alloys and stainless steels. With the pulsed current, avoiding the short circuiting, the relation power / wire speed increases because the voltage never reaches values lower than $15 \mathrm{~V}$. Then, the logic of control is just to avoid short circuits, hence yielding system configuration with more than one drop per period illogical. What is vital for the pulse technique is the physical arrangement of the wave variables. So, this article examines several sets of pulse current and pulse time, constituting different detachment parameters, and also different droplet diameters. The relationship between those variables is examined, considering the exponent of the current pulse equal to 2.3, according to researcher Amin, and the exponent 2.0, according to most adoptions in the literature. However, the value of 1.83 was found as the best fit for the wire ER4043. It was also shown that the best condition of detachment is when it occurs immediately after the pulse phase. Furthermore, it was shown that the stability coefficient $(\varphi)$ proposed by Gosh does not meet the proposed objectives.
\end{abstract}

Key-words: detachment parameter, detachment of droplets, aluminum welding.

\section{Introdução}

Apesar de ter surgido há mais de seis décadas, o processo de soldagem MIG/MAG continua a gerar sempre novas expectativas e tem sido continuamente assunto de pauta. Para um leigo, ao examinar o conjunto do processo, o mesmo se parece como nos seus primórdios. Entretanto, as inserções tecnológicas são de grande profusão e diversidade, restando apenas a similitude das partes macro componentes. Esta profusão

(Convidado em 15/05/2012; Texto final em 27/08/2012). de inovações tem como causa o aumento da importância que a tecnologia da soldagem obteve em relação aos demais processos de fabricação e o processo MIG/MAG é aquele que oferece as maiores chances de receber inovações. Entretanto, o que permitiu este desenvolvimento foram invenções em vários ramos da ciência e tecnologia, as quais envolvem a eletrônica de controle, a eletrônica de potência, a informática e os sistemas de acionamento. Em consequência, inúmeras empresas espalhadas pelo mundo lançam no mercado a cada ano um grande número de novidades, anunciando soluções e objetivos, que deixam o usuário com dificuldades de entendimento. De fato, as explicações para grande parte dos resultados obtidos não refletem adequadamente a realidade científica. As verdadeiras razões para que uma determinada tecnologia seja boa são mascaradas 
por frases de efeito mercadológico. Isto, para a ciência, se torna um desserviço grave e, por esta razão, os laboratórios e institutos de pesquisa devem exercer com bastante empenho sua ação. É neste contexto que se insere o presente trabalho, que se propõe a dar uma contribuição na análise da versão do processo MIG com corrente pulsada aplicada ao alumínio.

\section{Fundamentação}

Após a sua invenção nos idos de 1940, o processo MIG/ MAG permaneceu por muito tempo sendo utilizado com fontes comandadas em tensão, já que era a maneira possível para que o processo funcionasse na faixa onde a transferência metálica ocorre por curto-circuito. Nesta faixa de operação e para as fontes de soldagem convencionais, o arco não tem condições de permanecer aceso continuamente, uma vez que a velocidade de fusão não é suficiente para evitar o contato físico do eletrodo com a peça. Neste caso, a corrente não pode permanecer fixa, pois, do contrário, a gota metálica formada no extremo do eletrodo não se destaca, fazendo com que o eletrodo adira à peça e o arco não tenha condições de reacender. Desse modo, nos sistemas convencionais, a corrente tem de estar livre para variar a fim de que a cada curto-circuito o arco possa se restabelecer, propiciando a continuidade do processo. De qualquer maneira, o processo é conduzido com ocorrências de curtos-circuitos completamente inevitáveis. Estes curtos-circuitos, os quais ocorrem com grande restrição de controle, implicam em tensões elétricas muito baixas, produzindo uma relação potência/ velocidade de arame também baixa. Isto não é um problema grave na soldagem de aço carbono em geral, mas se torna crítico na soldagem de inoxidáveis e na soldagem de alumínio e suas ligas. Por este motivo foi desenvolvida a tecnologia da corrente pulsada, a qual nada mais é do que uma antecipação da elevação da corrente, antes que o curto-circuito aconteça e a tensão caia a níveis muito baixos. Nas primeiras fontes de energia lançadas no mercado com esta filosofia, esta elevação da corrente não era diretamente regulada, mas sim por intermédio da elevação da tensão. Então, de fato, as fontes de energia eram comandadas em tensão com a possibilidade de pulsação da mesma. Além disso, a frequência de pulsação não era livremente regulada, mas sim igual à frequência da rede de fornecimento de energia ou aos seus múltiplos e submúltiplos. Esta maneira de produzir pulsação nunca alcançou êxito prático. Foi somente com o advento das fontes de controle da corrente que o processo pulsado atingiu seus propósitos. Entretanto, após já mais de três décadas de utilização, o desenho da onda pulsada é ainda distinto de fabricante para fabricante. Permanecem ainda controvérsias sobre qual conjunto de variáveis é o mais adequado e, no caso da soldagem de ligas de alumínio, é onde isto se acentua mais fortemente. Muito se comenta sobre estabilidade do processo sem que haja um entendimento adequado sobre seu significado prático.

Sobre a sua base de funcionamento, apesar de algumas elucubrações sem sentido prático, é quase universalmente aceito que o adequado funcionamento da tecnologia de controle da transferência por corrente pulsada tem como pilar o destacamento de uma gota por período. Entretanto, permanece até hoje algumas dúvidas resultantes de discussões de vários pesquisadores e que algumas vezes são conflitantes entre alguns. Uma das questões se refere ao momento em que as gotas devem ser expelidas a fim de que seja obtida a melhor estabilidade do processo. Uma segunda questão discutida é a relação otimizada entre o valor da corrente de pulso e o seu respectivo tempo de duração. Pesquisadores, a exemplo de Gosh et al [1] [2], propõem índices de estabilidade que são muito mais uma aplicação matemática do que de fato uma interpretação física dos fenômenos. Em terceiro lugar viria a questão de qual seria o melhor diâmetro de gota, o que implica na frequência da transferência metálica, e se este diâmetro de gota deve ter correlação com a corrente de pulso e o seu tempo de duração. Por último vem a questão da estabilidade do comprimento do arco, fato que na soldagem de ligas de alumínio é revestido de tão alta importância, uma vez que oferecem muito maior dificuldade do que as ligas de aço. Esta é uma das razões para o surgimento de fontes de soldagem com as chamados controles adaptativos. Em razão de todas estas questões, o presente artigo se propõe a dar algumas respostas.

\section{Princípios Básicos do Controle da Transferência de Gotas por Corrente Pulsada}

Conforme já foi citado anteriormente, a corrente pulsada pode ser considerada como uma antecipação de uma elevação da corrente que normalmente ocorre quando a potência é baixa. Esta antecipação é para evitar o curto-circuito e assim obter alguns diferenciais em relação à soldagem com transferência convencional. Alguns destes diferenciais são: uma drástica redução da salpicagem, um aumento relativo da tensão elétrica para uma mesma velocidade de arame e assim a obtenção de uma geometria do cordão de solda com melhor adequação. Esta última característica é especialmente perceptível na soldagem de aços inoxidáveis devido à baixa capacidade de molhamento destas ligas e na soldagem de ligas de alumínio, que, por possuírem alta condutividade térmica, também causam pouco molhamento. Assim, comparada com a transferência por curtocircuito, a transferência pela utilização de corrente pulsada utiliza uma potência maior para a mesma velocidade de arame.

Dois critérios fundamentais devem ser atentados como premissa da seleção do conjunto de variáveis. Em primeira instância é que as variáveis elétricas selecionadas sejam tal que a velocidade do arame se equilibre com a velocidade de fusão do mesmo. O segundo critério é que a cada período corresponda uma gota transferida. Para o cumprimento do primeiro critério não há uma dependência crucial de variáveis especificas, já que todas influenciam de maneira idêntica. Já para o cumprimento do segundo critério, a corrente de pulso (Ip) e seu respectivo tempo (tp) são as variáveis determinísticas. Entretanto, a primeira é ainda mais relevante, sendo considerada pela maioria dos autores como em segundo grau em relação à segunda. Desse modo, segundo a literatura, é definida uma constante de destacamento de gota como $\mathrm{D}=\mathrm{Ip}^{2} \mathrm{x}$ tp. Os trabalhos de Amin consideram que a corrente de pulso possui um expoente igual a 2,3 [3] [4]. A restrição de seleção individual destas variáveis é tão somente quanto ao valor mínimo da corrente de pulso, que tem de ser superior ao limite superior da faixa de transição entre 
o curto-circuito convencional e a transferência goticular axial, designada convencionalmente como spray. Entretanto, embora o conjunto de variáveis Ip e tp possa atender ao requisito de uma gota por pulso, obedecendo a constante de destacamento, é constatado que nem a estabilidade da transferência e nem a solda obtida com cada par de variáveis são iguais.

Além desses aspectos globais de controle da transferência de uma gota por pulso, há aspectos muito específicos que vão às minúcias, como o instante temporal em que as gotas são transferidas. Existem proposições de que o destacamento ocorra no final da fase de pulso e proposições de destacamento no início da fase de base.

Adicionalmente, há um aspecto vital na soldagem de ligas de alumínio que transcende a estabilidade da transferência com muito maior relevância, pois pode produzir a interrupção do processo. Trata-se do desequilíbrio da velocidade de fusão do eletrodo em relação à sua velocidade de alimentação. Este desequilíbrio não ocorre quando a transferência metálica está na faixa do curto-circuito, conforme apresentado por Kiyohara [5], porque para uma determinada corrente de soldagem e uma velocidade de arame, há somente uma tensão média possível. Isto é visualmente verificável pela observação do diagrama cartesiano tensão x corrente para velocidades de arames fixas, pois a linha que representa uma mesma velocidade de arame é inclinada em relação aos dois eixos cartesianos. Entretanto, quando o mecanismo de transferência não mais é dependente da ocorrência de curtos, a referida linha é essencialmente vertical. Isto significa que diferentes tensões satisfazem o equilíbrio da velocidade de fusão. Idêntico comportamento ocorre também com aço, mas neste, o comprimento do arco fundamentalmente não é alterado porque existe uma resistividade apreciável do arame, o que não é o caso das ligas de alumínio. Por isso, há nessas ligas uma tendência de haver oscilações no comprimento do arco quando da utilização de fontes de corrente. Em consequência disso, vários fabricantes de fontes de soldagem anunciam que seus equipamentos são dotados de sistemas adaptativos, os quais previnem tal inconveniente. Não obstante, o modo como é estruturado o conjunto de variáveis, produzindo os destacamentos das gotas temporalmente distintos e ainda com diâmetros também distintos, pode influir na questão da citada instabilidade do comprimento do arco. Por isso, a metodologia a seguir foi programada para contribuir na demonstração destes fatos.

\section{Metodologia}

A análise foi restrita ao arame ER4043 de 1,2 mm, a uma corrente média fixa de $80 \mathrm{~A}$, com quatro conjuntos de variáveis de pulso, tentando obedecer a região de parâmetro de destacamento de uma gota por pulso [3], segundo a filosofia original da corrente pulsada. De acordo com Amin, o expoente de Ip deve ser 2,3, mas muitos autores [6] utilizam 2,0 como expoente. Considerando estes dois valores, as curvas correspondentes às referidas equações foram representadas na Figura 1 a fim de uma ampla visualização do campo possível de regulagem para obtenção de uma gota por período. Cada um dos quatro grupos de variáveis foi analisado para três diâmetros de gota (dg) diferentes: $1,10-1,20$ e 1,44 mm. Para o cálculo das variáveis da onda pulsada foi adotada a velocidade do arame de $4 \mathrm{~m} / \mathrm{min}$, a qual produzia um comprimento do arco de aproximadamente 5 mm para uma Distância do Bico de Contato até a Peça (DBCP) de $15 \mathrm{~mm}$. Assim, as variáveis resultaram, conforme a tabela 1 . Para a corrente de pulso de $160 \mathrm{~A}$ e tempo de base de 5,7 ms, fisicamente não é possível sua realização para diâmetro de gota de $1,1 \mathrm{~mm}$ porque resultaria numa corrente de base negativa. A avaliação da estabilidade dos procedimentos foi procedida por meio de depósito sobre chapa na posição plana e velocidade de soldagem fixada em $6,67 \mathrm{~mm} / \mathrm{s}(40 \mathrm{~cm} / \mathrm{min})$, com aquisição de dados para a elaboração de oscilogramas e ciclogramas e filmagem de alta velocidade. O índice $\varphi$ proposto por Gosh [1] [2] foi utilizado para análise com a pressuposição que o mesmo não representa o que a ele é atribuído.

Tabela 1. Conjunto de parâmetros de soldagem MIG-P, com cálculo de $\varphi$ e constante de destacamento de gotas (D)

\begin{tabular}{|c|c|c|c|c|c|c|c|c|c|}
\hline Grupo & Cód. & Ip (A) & tp (ms) & $\begin{array}{c}\text { dg }(\mathrm{mm}) \\
\text { projetada }\end{array}$ & Ib (A) & tb (ms) & $\varphi=(\mathbf{I b} / \mathbf{I p})^{*} \mathbf{f t b}$ & D (Ip $\left.{ }^{2} . t p\right)$ & $D\left(I p^{2,3} \cdot t p\right)$ \\
\hline \multirow{3}{*}{1} & 1 & 160 & 5,7 & 1,1 & -25 & 4,3 & - & \multirow{3}{*}{145920} & \multirow{3}{*}{668884} \\
\hline & 2 & 160 & 5,7 & 1,2 & 18 & 7,3 & 0,0632 & & \\
\hline & 3 & 160 & 5,7 & 1,44 & 53 & 16,8 & 0,2473 & & \\
\hline \multirow{3}{*}{2} & 4 & 300 & 2 & 1,1 & 25 & 8 & 0,0667 & \multirow{3}{*}{180000} & \multirow{3}{*}{996343} \\
\hline & 5 & 300 & 2 & 1,2 & 40 & 11 & 0,1128 & & \\
\hline & 6 & 300 & 2 & 1,44 & 59 & 20,5 & 0,1792 & & \\
\hline \multirow{3}{*}{3} & 7 & 350 & 0,8 & 1,1 & 57 & 9,2 & 0,1498 & \multirow{3}{*}{98000} & \multirow{3}{*}{568128} \\
\hline & 8 & 350 & 0,8 & 1,2 & 62 & 12,2 & 0,1662 & & \\
\hline & 9 & 350 & 0,8 & 1,44 & 70 & 21,7 & 0,1929 & & \\
\hline \multirow{3}{*}{4} & 10 & 350 & 1,2 & 1,1 & 37 & 8,8 & 0,0930 & \multirow{3}{*}{147000} & \multirow{3}{*}{852193} \\
\hline & 11 & 350 & 1,2 & 1,2 & 53 & 10,8 & 0,1363 & & \\
\hline & 12 & 350 & 1,2 & 1,44 & 65 & 21,3 & 0,1758 & & \\
\hline
\end{tabular}




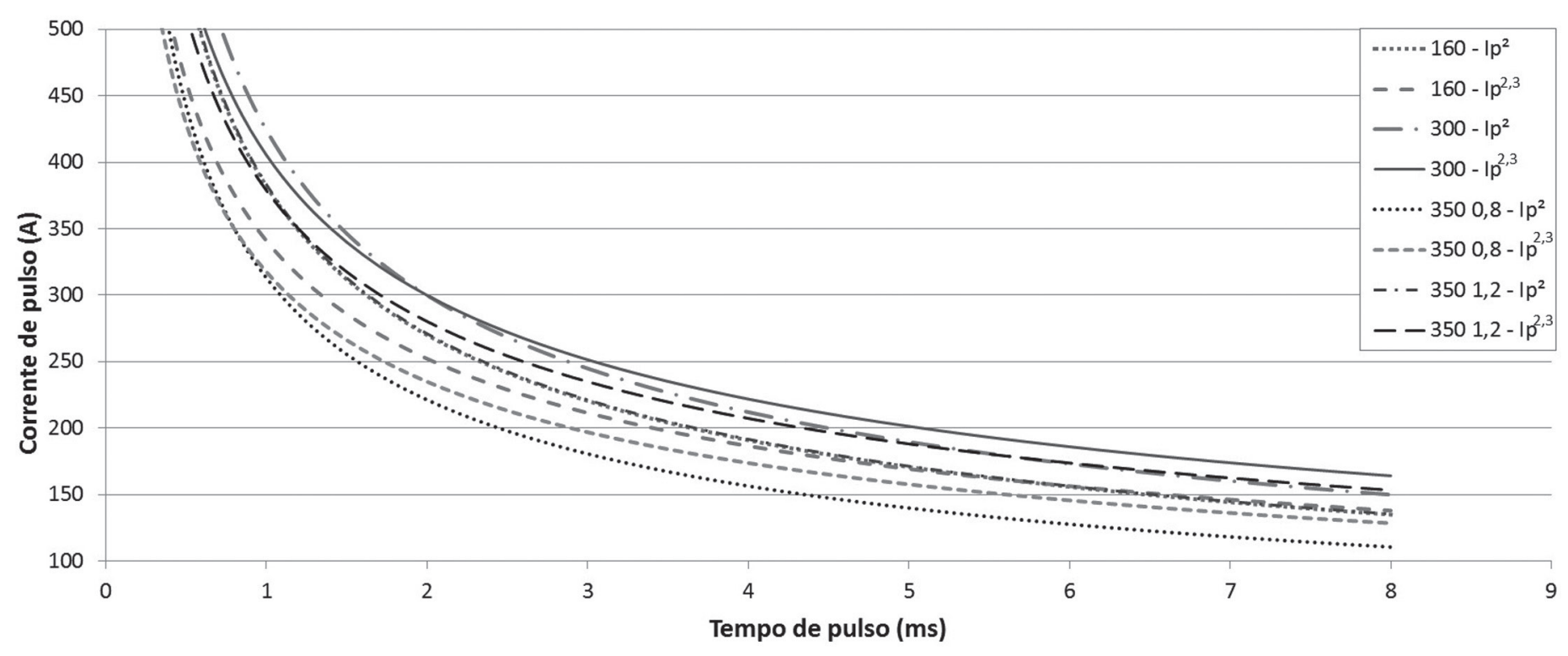

Figura 1. Curvas representativas de parâmetros de destacamento de gota
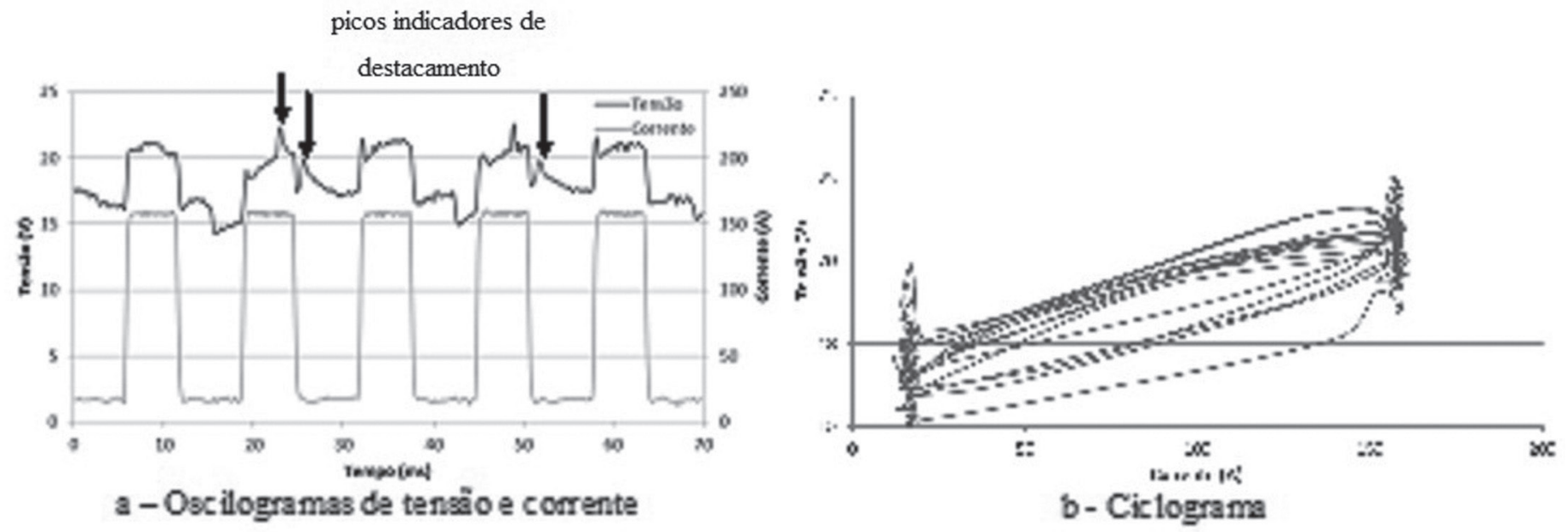

Figura 2. Aquisição de dados referentes ao depósito de solda com $\mathrm{Ip}=160 \mathrm{~A}$, tp=5,7 ms, $\mathrm{Ib}=18 \mathrm{~A}$, tb=7,3 ms e diâmetro de gota de $1,2 \mathrm{~mm}$

\section{Resultados e discussões}

Como já foi mencionado, o primeiro conjunto de variáveis (cód.1) resulta em uma impossibilidade física de execução. O segundo conjunto (cód.2) resultou nos oscilogramas da Figura 2a e no ciclograma da Figura 2b. O depósito obtido é o apresentado na Figura 3. A análise da filmagem revela que a transferência de gotas acontece de forma bastante irregular, ocorrendo momentos em que a gota destaca no pulso e outros na base, aparecendo ainda situações de duas gotas por pulso e até mesmo pulsos de corrente que não destacam nenhuma gota. $\mathrm{O}$ oscilograma de tensão mostra tal irregularidade, com pulsos de destacamento em diferentes instantes. O ciclograma (Figura 2b) é outro testemunho da irregularidade.

É notório que não existe uma boa repetitividade, apesar do depósito de solda apresentar uma aparência razoável com textura lisa. Conforme comparações futuras, a causa disto pode ser atribuida a pequena velocidade das gotas em razão delas serem expelidas em corrente relativamente baixa. $\mathrm{O}$ fator $\varphi$ foi $\mathrm{o}$ menor de todos os conjuntos.

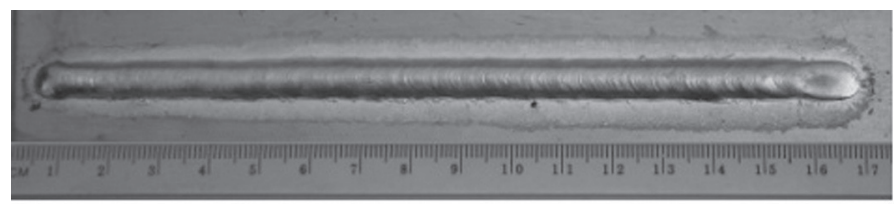

Figura 3. Depósito de solda referente aos dados da Figura 2.

O terceiro conjunto de variáveis (cód.3) resultou nos oscilogramas da Figura 4a e foi constatado que as gotas foram destacadas sempre no pulso. Pela observação do ciclograma, 


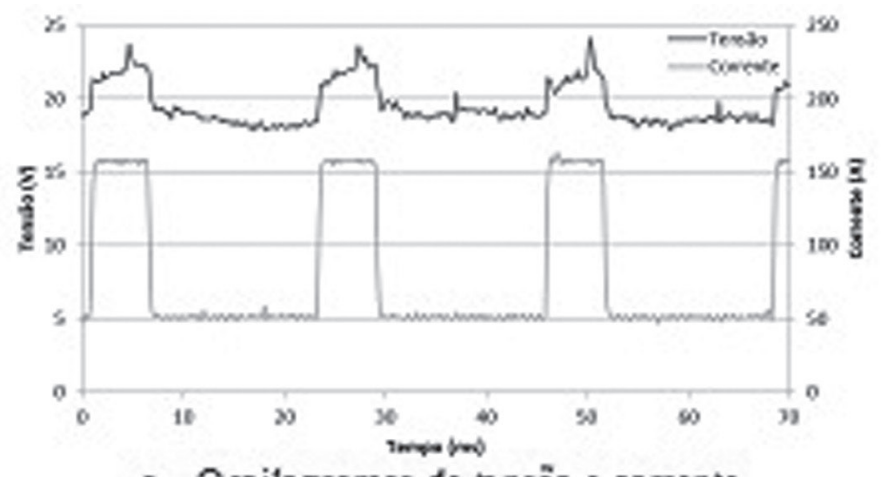

a - Oscilogramas de tensão e corrente

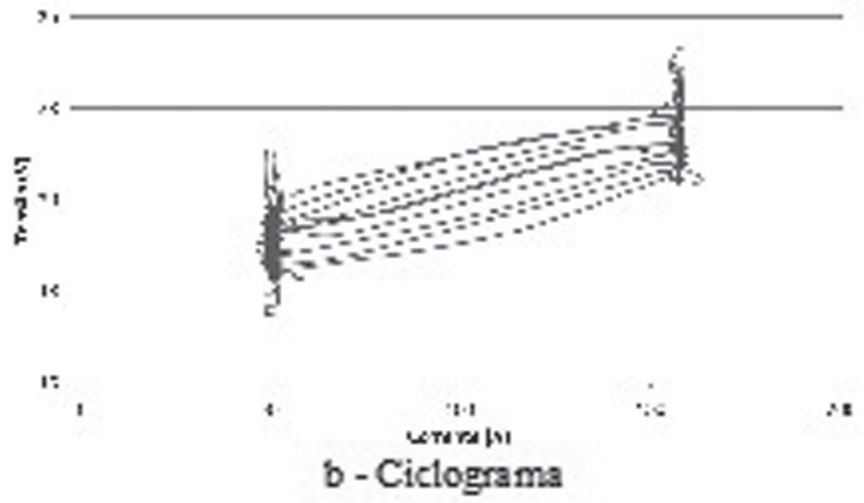

Figura 4. Aquisição de dados referentes ao depósito de solda com $\mathrm{Ip}=160 \mathrm{~A}, \mathrm{tp}=5,7 \mathrm{~ms}, \mathrm{Ib}=53 \mathrm{~A}$ e tb=16,8 ms e diâmetro de gota de $1,44 \mathrm{~mm}$.

a estabilidade do procedimento foi melhor do que o anterior, apesar de um fator $\varphi$ maior. O depósito de solda resultante (Figura 5) também revela um melhor acabamento. O ciclograma (Figura 4b) é também menos disperso.

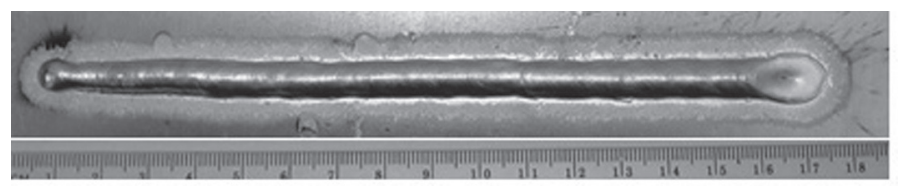

Figura 5. Cordão de solda referente aos dados da Figura 4.

O segundo grupo de ensaios, que possui como parâmetros de pulso uma corrente de 300 A com um tempo de $2 \mathrm{~ms}$, sendo composto pelos conjuntos cód.4, cód.5 e cód.6, resultou em cordões com baixa qualidade no acabamento e com o aparecimento de alguns salpicos finos. Os sinais oscilográficos revelam uma razoável estabilidade. Entretanto, a visualização do momento de destacamento das gotas não apareceu de forma evidente nos oscilogramas de tensão. Utilizando-se da filmagem em alta velocidade foi detectado que a gota é destacada durante o momento do pulso e que a quantidade de energia sobressaliente promove a continuidade da fusão do arame que, em algumas situações, ocasiona o destacamento de uma segunda gota com menor diâmetro (Figura 6b). Quando essa segunda gota não é expelida ocorre um efeito repulsivo tipo "chicote" com o material fundido na ponta do arame (Figura 6a), originando os já referidos salpicos finos. Dos três conjuntos de variáveis deste grupo, o de código 4 também apresenta um fator $\varphi$ baixo, sendo o segundo na ordem crescente.

Em todos os três conjuntos, cód.7, cód.8 e cód.9, contidos no terceiro grupo de ensaios, com $\mathrm{Ip}=350 \mathrm{~A}$ e tp=0,8 ms, o destacamento da gota aconteceu no início do tempo de base. Quando o diâmetro de gota teórico foi de $1,1 \mathrm{~mm}$, ou seja, conjunto cód.7, apesar do oscilograma ter demonstrado um comportamento estável, foi verificada na filmagem a formação de respingos em consequência de um efeito repulsivo sobre o material fundido imediatamente após o destacamento da gota (Figura 7).

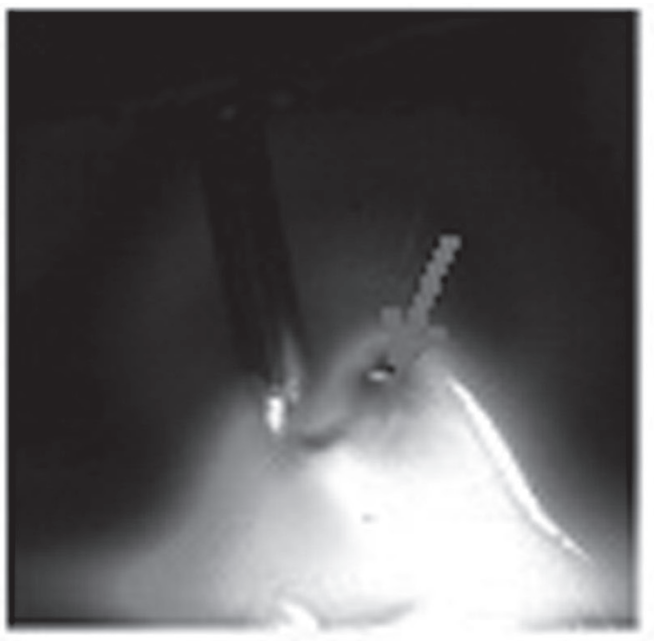

a

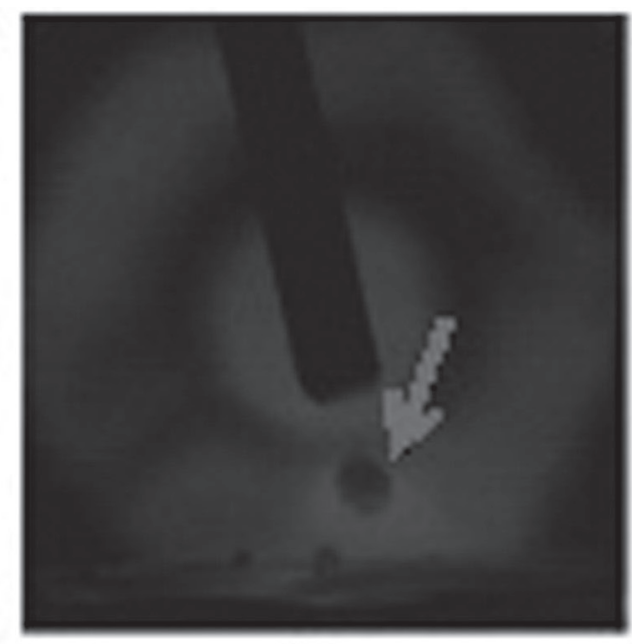

b

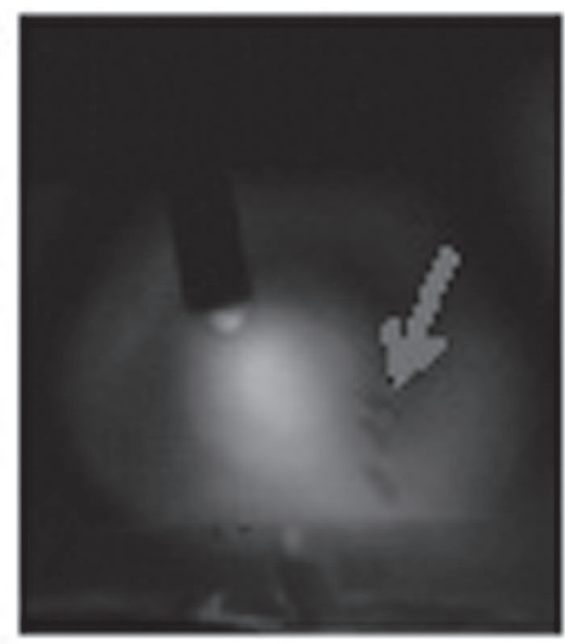

C

Figura 6. Comportamento da transferência metálica para o segundo grupo de ensaios ( $\mathrm{Ip}=300 \mathrm{~A} \mathrm{e} \mathrm{tp=2} \mathrm{ms);}$

a - Efeito repulsivo para o conjunto de parâmetros com diâmetro de gota $1,1 \mathrm{~mm} ; \mathrm{b}$ - Destacamento da segunda gota no conjunto para $\mathrm{dg}=1,2 \mathrm{~mm}$; $\mathrm{c}-$ Formação de respingos no conjunto com $\mathrm{dg}=1,44$. 


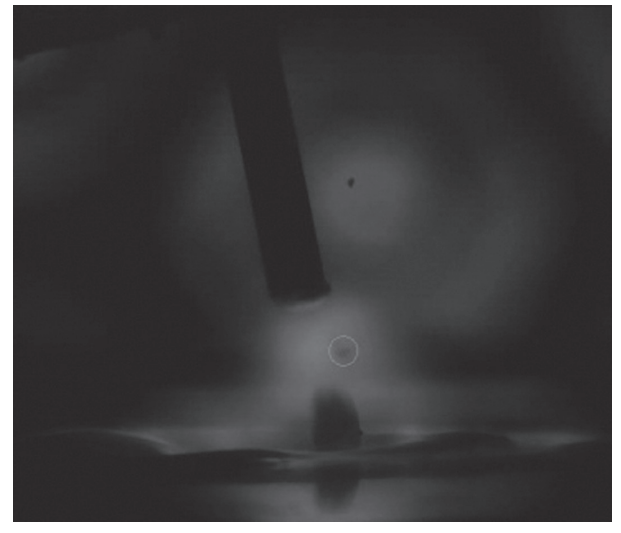

Figura 7. Destacamento de gota para o conjunto cód.7 com Ip $=350 \mathrm{~A}, \mathrm{tp}=0,8 \mathrm{~ms}, \mathrm{Ib}=57 \mathrm{~A}$ e tb $=9,2 \mathrm{~ms}$ para diâmetro de gota de $1,1 \mathrm{~mm}$.

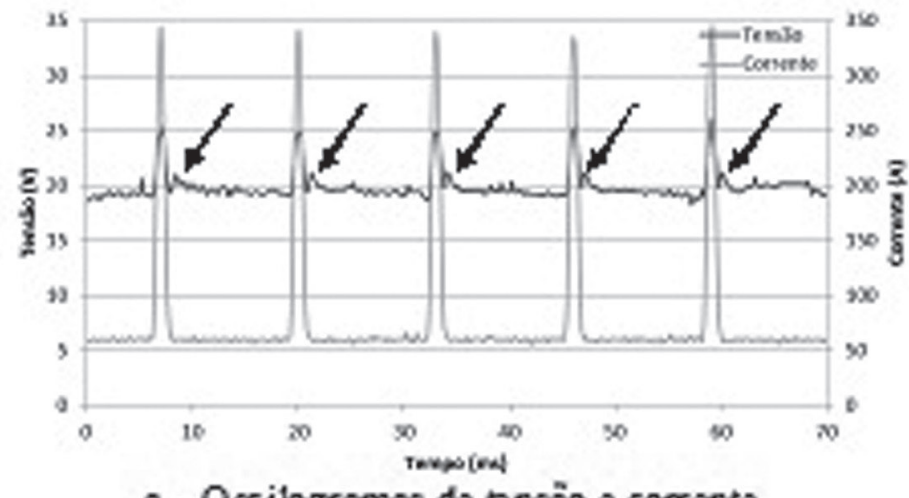

a - Oscilogramas de tensāo e corrente
Com o aumento do diâmetro de gota para $1,2 \mathrm{~mm}$, a tensão de base permaneceu bastante estável e houve somente uma gota em cada início da fase de base (Figura 8). O depósito de solda resultou em uma textura lisa como no caso do conjunto 2 (160 A e 5,7 ms). A razão para isto pode ser atribuída à menor velocidade das gotas, pois foram expelidas na fase de base (62 A). $\mathrm{O}$ valor de $\varphi$ foi relativamente elevado, o que revela a sua não utilidade.

Os picos de tensão referentes ao momento do destacamento de gota foram bem maiores para 1,44 $\mathrm{mm}$ de diâmetro de gota (Figura 10). Isto pode contribuir com certo grau da instabilidade, associada a um tempo de base também muito grande.

Figura 8. Aquisição de dados referentes ao depósito de solda com Ip $=350 \mathrm{~A}, \mathrm{tp}=0,8 \mathrm{~ms}, \mathrm{Ib}=62 \mathrm{~A}$ e tb $=12,2 \mathrm{~ms}$ e diâmetro de gota de $1,2 \mathrm{~mm}$.

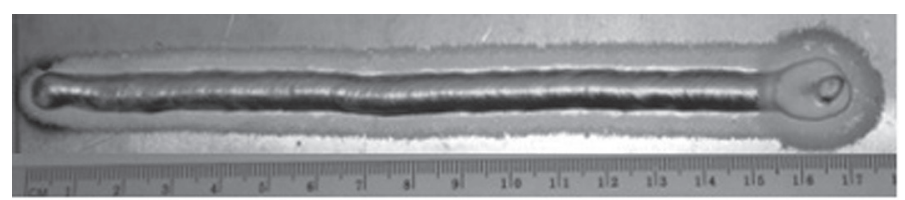

Figura 9. Depósito de solda referente à Figura 8.

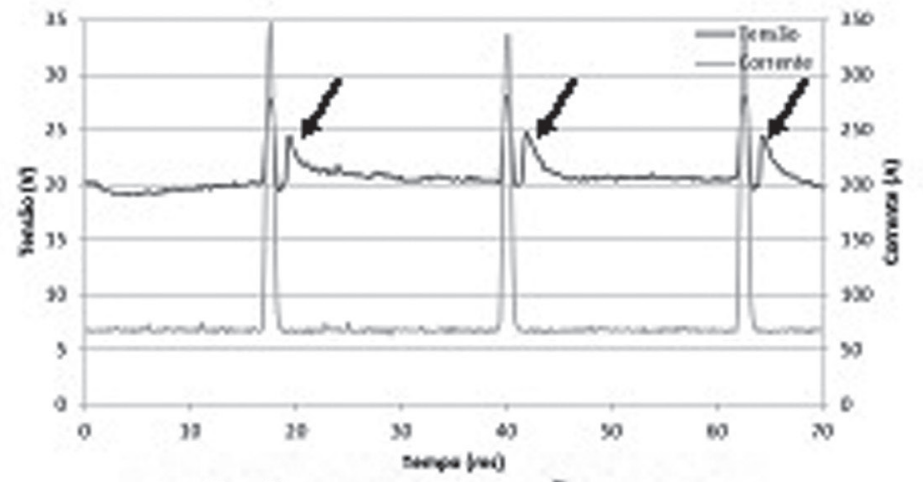

a - Oscilogramas de tensāo e corrente
Para os conjuntos cód.10, cód.11e cód.12, com corrente de 350 A e tempo de pulso de $1,2 \mathrm{~ms}$, foi constatado que o destacamento de gota acontece durante o pulso de corrente. Como já ocorreu para os outros casos com o diâmetro de gota de 1,1 mm, não há no oscilograma de tensão um indicativo da transferência. Esta só é confirmada com a filmagem de alta velocidade. Já para diâmetro de gota de 1,2 mm, reapareceram

Figura 10. Aquisição de dados referentes ao depósito de solda com $\mathrm{Ip}=350 \mathrm{~A}, \mathrm{tp}=0,8 \mathrm{~ms}, \mathrm{Ib}=70 \mathrm{~A}$ e tb $=21,7 \mathrm{~ms}$ e diâmetro de gota de $1,44 \mathrm{~mm}$. 


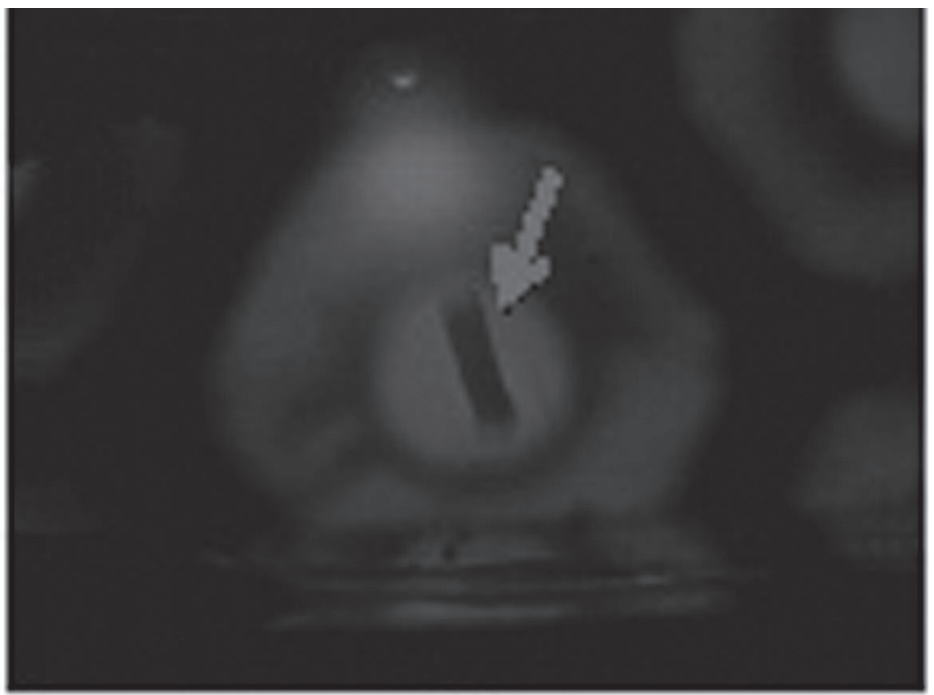

a

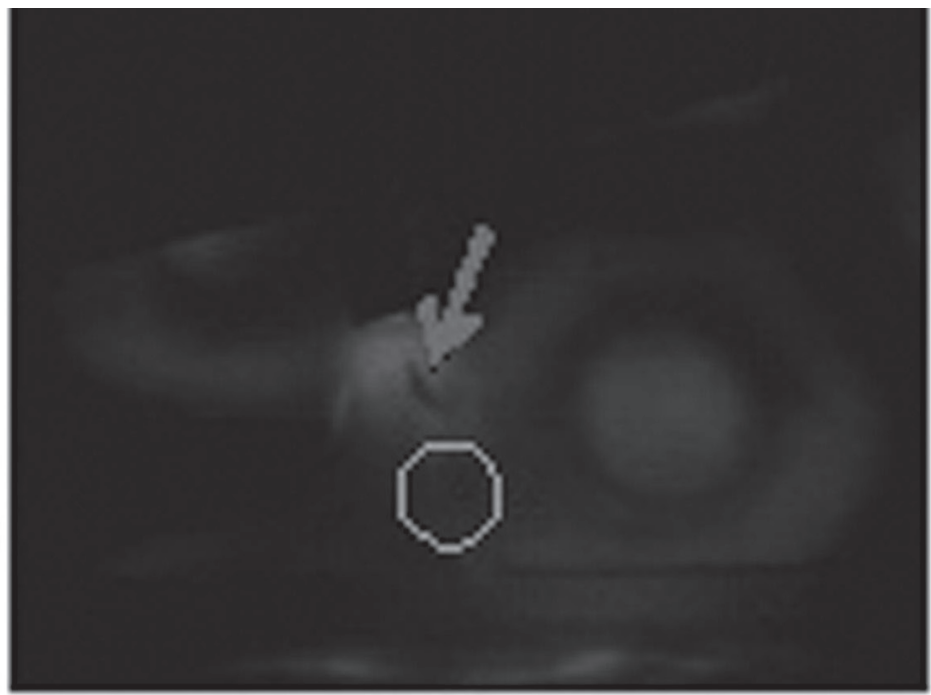

b

Figura 11. Comportamento da transferência metálica ( $\mathrm{Ip}=350 \mathrm{~A} ; \mathrm{tp}=1,2 \mathrm{~ms}$ ); a - Alongamento da gota no conjunto 11 com dg $1,2 \mathrm{~mm}$; $\mathrm{b}$ - Formação de respingos no conjunto $12 \mathrm{com} \mathrm{dg}=1,44$

no oscilograma de tensão os picos relativos à transferência. $\mathrm{O}$ destacamento da gota em um alto nível de corrente provocou um alongamento da gota (Figura 11a). Para diâmetro de gota de 1,44 mm há novamente uma forte influência sobre os picos de tensão no respectivo oscilograma e que somada ao grande tempo de base, produz uma forte instabilidade do processo. A filmagem realizada com esse parâmetro mostra a formação de respingos logo após o destacamento da gota (Figura 11b). Em todos os conjuntos desse grupo, o depósito de solda ficou com aparência de que houve uma forte pressão do arco, como pode ser observado para o caso do conjunto cód.10 na Figura 12.

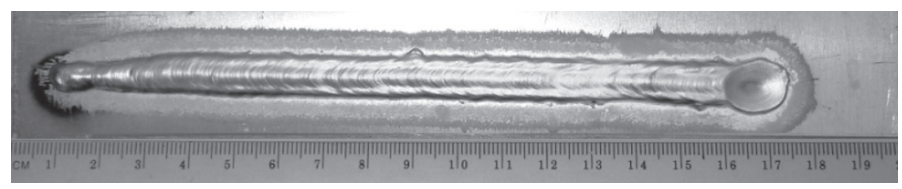

Figura 12. Depósito de solda depósito de solda com $\mathrm{Ip}=350$ $\mathrm{A}, \mathrm{tp}=1,2 \mathrm{~ms}, \mathrm{Ib}=37 \mathrm{~A}$ e tb $=8,8 \mathrm{~ms}$ e diâmetro de gota de 1,1 $\mathrm{mm}$
De todos os conjuntos de variáveis, o que apresentou melhores resultados foi o oitavo (cód. $8, \mathrm{Ip}=350 \mathrm{~A}$, tp=0,8 ms e $\mathrm{dg}=1,2 \mathrm{~mm}$ ). Aplicando os respectivos valores do parâmetro de destacamento (D) para uma corrente de pulso de 240 A (valor adotado por uma das mais famosas empresas fabricantes de fontes de soldagem), resulta em $\mathrm{tp}=1.9 \mathrm{~ms}$ para o expoente 2,3 de Ip e 1,7 ms para o expoente 2,0. Foram procedidos ensaios respectivos a estas duas condições, obedecendo aos dados da tabela 2. Os resultados revelaram boas condições de soldagem, mas não superiores à condição de $350 \mathrm{~A}$ e $0,8 \mathrm{~ms}$. Analisando os oscilogramas foi constatado que para esses dois conjuntos, numerados como 13 e 14 na tabela 2, o destacamento da gota aconteceu durante o pulso de corrente. Diminuindo o tempo de pulso para 1,6 ms observou-se que o destacamento ocorreu no início do tempo de base, a exemplo do conjunto 8 .

Tabela 2. Conjunto de variáveis de soldagem MIG-P, segundo o valor de $\mathrm{Ip}=240$ A proposto por um fabricante de fontes de soldagem com cálculo de $\varphi$

\begin{tabular}{|c|c|c|c|c|c|c|c|c|c|}
\hline Grupo & Cód. & $\mathbf{I p}(\mathbf{A})$ & $\mathbf{t p}(\mathbf{m s})$ & $\begin{array}{c}\mathbf{d g}(\mathbf{m m}) \\
\mathbf{p r o j e t a d a}\end{array}$ & $\mathbf{I b}(\mathbf{A})$ & $\mathbf{t b}(\mathbf{m s})$ & $\boldsymbol{\varphi}=(\mathbf{I b} / \mathbf{I p}) * \mathbf{f t b}$ & $\mathbf{D}\left(\mathbf{I} \mathbf{p}^{2} \cdot \mathbf{t p}\right)$ & $\mathbf{D}\left(\mathbf{I} \mathbf{p}^{\mathbf{2} . \mathbf{3}} \cdot \mathbf{t p}\right)$ \\
\hline \multirow{2}{*}{$\mathbf{5}$} & $\mathbf{1 3}$ & 240 & 1,9 & 1,2 & 53 & 11,1 & 0,1887 & 109440 & 566552 \\
\cline { 2 - 10 } & $\mathbf{1 4}$ & 240 & 1,7 & 1,2 & 56 & 11,3 & 0,2031 & 97920 & 506915 \\
\cline { 2 - 10 } & $\mathbf{1 5}$ & 240 & 1,6 & 1,2 & 58 & 11,4 & 0,2121 & 92160 & 477096 \\
\hline
\end{tabular}



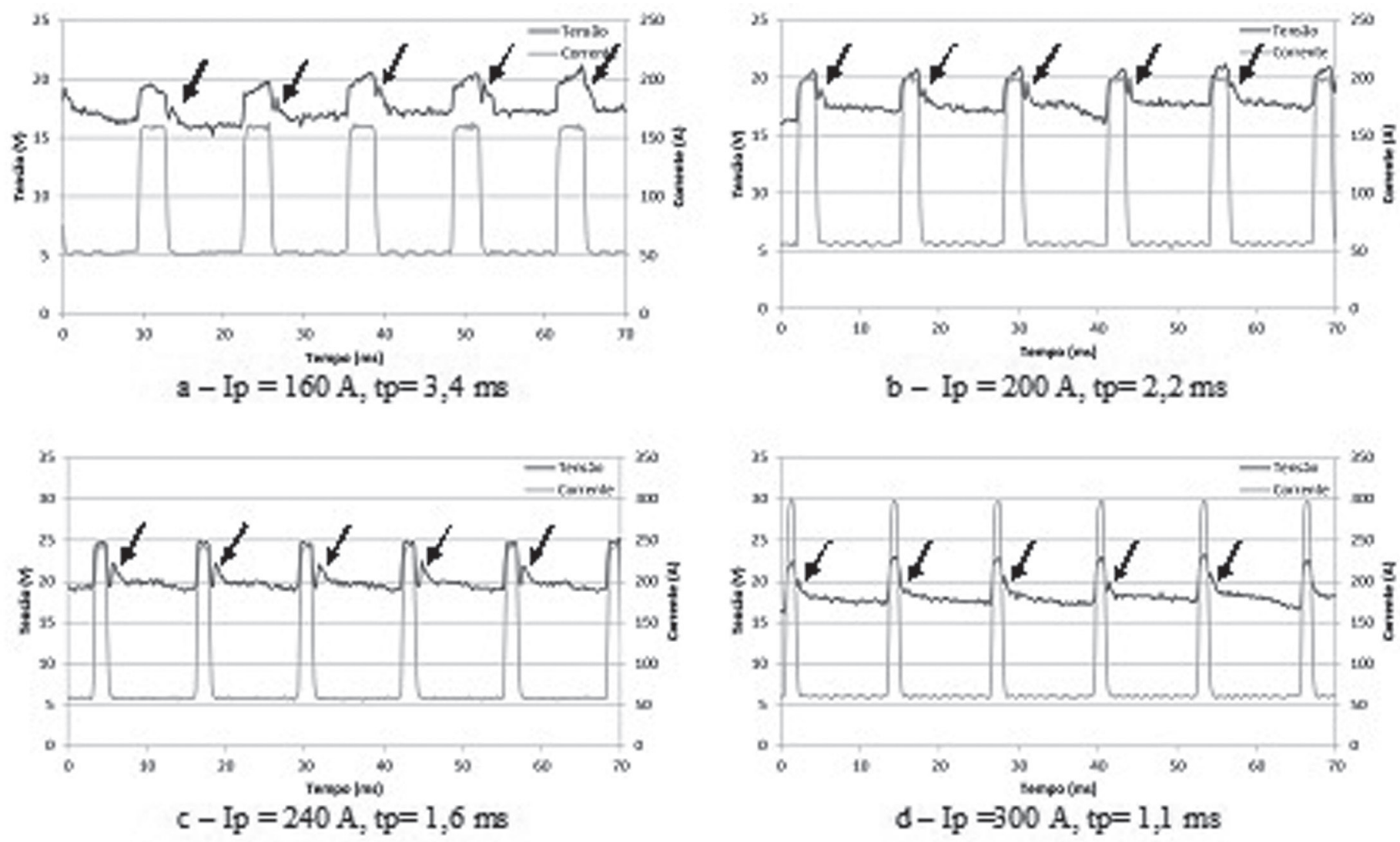

Figura 13. Aquisição de dados referentes aos depósitos para validação da relação $\mathrm{Ip}^{1,83} \cdot \mathrm{tp}=\mathrm{D}$

Com os dois pontos de corrente e tempo de pulso onde o destacamento de gota aconteceu no início do tempo de base foi possível determinar um novo expoente para a relação $\operatorname{Ip}^{x}$. tp $=\mathrm{D}$, uma vez que a constante de destacamento para esses dois pontos seria, teoricamente, igual. A fim de validar o novo valor de 1,83 encontrado para o expoente de Ip, foram obtidos os valores de tempo de pulso para as correntes de $160 \mathrm{~A}, 200 \mathrm{~A}$ e $300 \mathrm{~A}$, sendo 3,4 ms, 2,2 ms e 1,1 ms, respectivamente. Em uma nova sequência de ensaios sob esses parâmetros de pulso e considerando o $\mathrm{dg}=1,2 \mathrm{~mm}$, foi constatado que, em todos os casos, o destacamento da gota ocorreu logo no início da base, como colocado na Figura 13.

Ainda que haja uma correta seleção das variáveis que compõem a onda pulsada, como aqui descrito, propiciando o maior grau de repetitividade, a estabilidade com relação à manutenção do comprimento do arco não é absoluta, mesmo que melhor do que nos outros casos. Por isso, foi aplicado um controle adaptativo baseado na leitura da tensão de base, já que os autores deste trabalho estão de acordo com o proposto por Zhiming e Al-Erhayem [7]. Estes autores afirmam que as variações de resistência do arco, dissipação térmica e resistência no contato pouco influenciam a precisão da medição, sugerindo assim que a aquisição seja feita no tempo de base. Por outro lado, Essers e Gompel [8] citam que devido à grande quantidade de emissão de elétrons durante elevados valores de corrente, a leitura de tensão durante este período representaria melhor o comprimento do arco.
A metodologia aplicada para realizar o controle da altura do arco consiste em medir a tensão durante o tempo de base e comparar com uma referência fornecida pelo usuário. De acordo com o erro, o sistema atua no valor da corrente de base. A redução nos valores de tensão lidos durante a base corresponde a uma redução no comprimento do arco, ou seja, uma necessidade de aumentar a fusão do eletrodo para que seja mantido o valor de referência regulado pelo operador. No exemplo da Figura 14 o referido valor foi de 20,5 V, que é a tensão média, obtida do tempo de base no oscilograma da Figura 13c. Percebe-se claramente no exemplo a variação dos níveis da corrente de base durante a oscilação ocorrida no sinal de tensão.

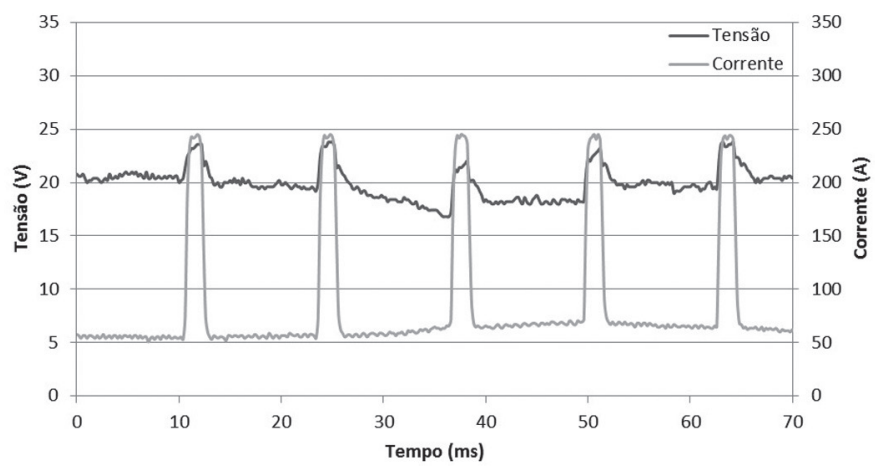

Figura 14. Oscilograma de tensão e corrente que mostra a atuação do sistema adaptativo de controle da altura do arco 
O resultado foi excelente, não havendo alterações do comprimento do arco, seja por causas fortuitas, seja por causa intencional, como em alguns ensaios onde a variação de DBCP aconteceu de $15 \mathrm{~mm}$ a $28 \mathrm{~mm}$.

\section{Conclusões}

Para o estabelecimento das relações entre as variáveis que compõem a forma de onda é fundamental a preocupação na obtenção de um destacamento de gota temporalmente repetitivo e que ocorra em correntes não muito elevadas. Foi constatado que, para o caso da liga de alumínio utilizada, o diâmetro de gota mais adequado é igual ao do diâmetro do eletrodo. Uma questão fundamental, que pode ser diretamente visualizada na tabela 1 , é que conjuntos de variáveis de destacamento com correntes de pulso baixas limitam o valor da corrente média mínima e isto é agravado para diâmetros de gota muito pequenos, que, no presente caso, nem viabilizaram matematicamente o primeiro conjunto de variáveis. O conjunto de variáveis de número 8 , com a utilização de corrente de pulso de $350 \mathrm{~A}$ e tempo de pulso de $0,8 \mathrm{~ms}$ propiciou a melhor estabilidade e ainda permite que se obtenham correntes médias extremamente baixas, porque em 80 A médios o valor da corrente de base foi de $62 \mathrm{~A}$, dando uma margem bastante grande de sua redução a fim de que se obtenha a redução da corrente média. Os resultados obtidos com a condição de $\mathrm{Ip}=240 \mathrm{~A}$ e tp=1,6 ms, possuem características visualmente semelhantes ao do conjunto 8. Entretanto, este último propicia uma maior rigidez do arco e ainda a já referida maior capacidade de redução nos valores de corrente média.

Quanto à questão do fator $\varphi$, foi evidenciado que ele não demonstra coerência com os resultados obtidos e, portanto, não oferece utilidade.

\section{Referências Bibliográficas}

[1] GHOSH, P. K. et al. Arc characteristics and behaviour of metal transfer in pulsed current GMA welding of aluminium alloy. Journal of Materials Processing Technology, 194, novembro 2007. 163-175.

[2] GHOSH, P. K. et al. Thermal and metal transfer behaviours in pulsed current gas metal arc weld deposition of Al-Mg alloy. Science and Technology of Welding and Joining, 11, março 2006. 232-242.

[3] AMIN, M. Pulse current Parameters for arc stability and controlled metal transer in arc welding. Metal Construction, 15 (5), May 1983. 272-278.

[4] AMIN, M. Synergic pulse MIG welding. Metal Construction, 13 (6), June 1981. 349-353.

[5] KIYOHARA, M.; YAMAMOTO, H.; HARADA, S. Melting characteristics of a wire electrode in the MIG-weldng of aluminum. Arc Physics and Weld Pool Behaviour, 1979. p. 165-175.

[6] DUTRA, J. C.; OLLÉ, L. F.; GOHR, R. J. O processo MIG/ MAG Pulsado com pulsação térmica. XXI Encontro Nacional de Tecnologias de Soldagem. Caxias do Sul, p. 889-902. 1995. [7] ZHIMING, O.; AL-ERHAYEM, O. Feedbacksteuerung zum metall-schutzgasschwei en mit impulslichtbogen. Schweißen und Schneiden, 41, 1989.

[8] ESSERS, W. G.; GOMPEL, M. R. M. V. Arc Control with Pulsed GMAW Welding. Welding Journal, USA, June 1984. 2632. 